Journal of Engineering Science and Technology Review 8 (2) (2015) 157 - 173 Special Issue on Synchronization and Control of Chaos: Theory, Methods and Applications

Research Article

\title{
Memristor: A New Concept in Synchronization of Coupled Neuromorphic Circuits
}

\author{
Ch. K. Volos ${ }^{*}$, , I. M. Kyprianidis ${ }^{1}$, I. N. Stouboulos ${ }^{1}$, E. Tlelo-Cuautle ${ }^{2}$ and S. Vaidyanathan ${ }^{3}$ \\ ${ }^{1}$ Department of Physics, Aristotle University of Thessaloniki, Thessaloniki, GR-54124, Greece. \\ ${ }^{2}$ Department of Electronics, INAOE, Luis Enrique Erro No. 1, 72840, Tonantzintla, Puebla, Mexico. \\ ${ }^{3}$ Research and Development Centre, Vel Tech University, Avadi, Chennai-600062, Tamil Nadu, India.
}

Received 2 September 2014; Revised 4 October 2014; Accepted 25 October 2014

\begin{abstract}
The existence of the memristor, as a fourth fundamental circuit element, by researchers at Hewlett Packard (HP) labs in 2008, has attracted much interest since then. This occurs because the memristor opens up new functionalities in electronics and it has led to the interpretation of phenomena not only in electronic devices but also in biological systems. Furthermore, many research teams work on projects, which use memristors in neuromorphic devices to simulate learning, adaptive and spontaneous behavior while other teams on systems, which attempt to simulate the behavior of biological synapses.

In this paper, the latest achievements and applications of this newly development circuit element are presented. Also, the basic features of neuromorphic circuits, in which the memristor can be used as an electrical synapse, are studied. In this direction, a flux-controlled memristor model is adopted for using as a coupling element between coupled electronic circuits, which simulate the behavior of neuron-cells. For this reason, the circuits which are chosen realize the systems of differential equations that simulate the well-known Hindmarsh-Rose and FitzHugh-Nagumo neuron models. Finally, the simulation results of the use of a memristor as an electric synapse present the effectiveness of the proposed method and many interesting dynamic phenomena concerning the behavior of coupled neuron-cells.
\end{abstract}

Keywords: Chaos, neuron, synapse, memristor, FitzHugh-Nagumo model, Hindmarsh-Rose model, complete syncronization.

\section{Introduction}

Until the beginning of 70ies the electronic circuit theory has been spinning around the three known, fundamental twoterminal circuit elements, which are known as: resistor $(R)$, capacitor $(C)$ and inductor $(L)$. These elements reflect the relations between pairs of the four electromagnetic quantities of charge $(q)$, current $(i)$, voltage $(v)$ and magnetic flux $(\varphi)$ that mathematically can be written as:

$$
\begin{aligned}
\mathrm{d} v & =R(i) \mathrm{d} i \\
\mathrm{~d} q & =C(v) \mathrm{d} v \\
\mathrm{~d} \varphi & =L(i) \mathrm{d} i
\end{aligned}
$$

In the case that the factors $C, L$ and $R$ have constant values, the corresponding circuit elements are linear. However, as it can be derived, a relation between the charge $(q)$ and the flux $(\varphi)$ is missing.

At that time (1971), Professor Leon Chua from the University of California at Berkley, dubbed this missing link by introducing the fourth fundamental element based on the symmetry arguments [1]. This fourth circuit element was named memristor $(M)$, an acronym for memory resistor, which its existence was conjectured due to the following missing relation between the charge $(q)$ and the flux $(\varphi)$ (Fig.1(a)).

$\mathrm{d} \varphi=M(q) \mathrm{d} q$

The multiplicative term $M(\bullet)$ is called the memristance function.

Dividing both sides of (2) by $\mathrm{d} t$ one obtains

$v=M(q) i$

If $M$ is constant, equation (3) is nothing but the defining relation of a linear resistor $(R)$, as it can be shown from (1).

* E-mail address: volos@physics.auth.gr ISSN: 1791-2377 (C) 2015 Kavala Institute of Technology. All rights reserved. 


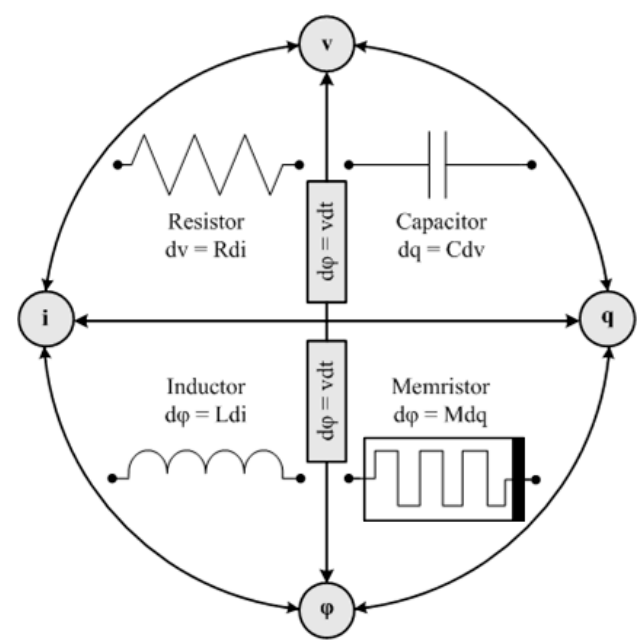

(a)

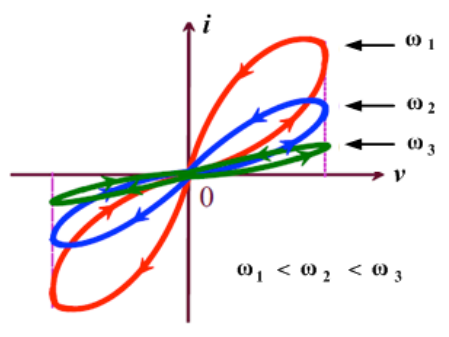

(b)

Fig. 1. The four circuit variables connected by the fundamental circuit elements and (b) a typical $v-i$ characteristic curves of a memristor driven by a sinusoidal voltage input.

However, Chua has proved theoretically that a memristor is a nonlinear element because its $v-i$ characteristic is similar to that of a Lissajous pattern. So, a memristor with a nonconstant $M$ describes a resistor with a memory, more precisely a resistor whose resistance depends on the amount of charge that has passed through the device.

A typical response of a memristor to a sinusoidal input is depicted in Fig.1(b). The 'pinched hysteresis loop currentvoltage characteristic' is an important fingerprint of a memristor. If any device has a current-voltage hysteresis curve, then it is either a memristor or a memristive device. Another signature of the memristor is that the 'pinched hysteresis loop' shrinks with the increase in the excitation frequency. The fundamentality of the memristor can also be deduced from this figure, as it is impossible to make a network of capacitors, inductors and resistors with an $v-i$ behavior forming a pinched hysteresis curve [2]. So, it will be very easy to visualize the inevitable presence of the memristor, if we rewrite equations (1) \& (2) as shown in the Table 1.

As we can see from Table 1 the integral can be used in four different ways to describe the relationship between current and voltage by either using it or not using it. We note that the equations for resistance and memristance appear identical, except for the presence of the integral sign in the latter's case on both sides of laws. However, this integral cannot be cancelled because the constant of integration need not be zero. And this is the constant that makes the memristor 'remember' the previous state. [1]:

Some of the more interesting properties of memristor are

- Non-linear relationship between current $(i)$ and voltage $(v)$

- Does not store energy.

- Similar to classical circuit elements, a system of memristors can also be described as a single memristor.

- Reduces to resistor for large frequencies as evident in the $v-i$ characteristic curve.

- Memory capacities based on different resistances produced by the memristor.

- Non-volatile memory possible if the magnetic flux and charge through the memristor have a positive relationship $(M>0)$.

Furthermore, a more generalized class of systems, in regard to the original definition of a memristor, called memristive systems [2], is introduced. An $n$ th-order currentcontrolled memristive one-port is represented by

$$
\begin{aligned}
& v=R(w, i, t) i \\
& \frac{\mathrm{d} w}{\mathrm{~d} t}=f(w, i, t)
\end{aligned}
$$

where $w \in \mathrm{R}^{n}$ is the $n$-dimensional state variable of the system.

Also, the $n$ th-order voltage-controlled memristive oneport is defined as:

$$
\begin{aligned}
& i=G(w, v, t) v \\
& \frac{\mathrm{d} w}{\mathrm{~d} t}=f(w, i, t)
\end{aligned}
$$

Similarly to memristor, a memristive system has the following properties:

The memristive system should have a dc characteristic curve passing through the origin.

- For any periodic excitation the $v-i$ characteristic curve should pass through the origin.

- As the excitation frequency increases toward infinity the memristive system has a linear behavior.

- The small signal impedance of a memristive system can be resistive, capacitive, or inductive depending on the operating bias point.

After Chua's work in 1971, only a few works appeared in the literature for a long time since it was thought that the memristor was only a theoretical element and it could not be realized practically. 
Ch. K. Volos, I. M. Kyprianidis, I. N. Stouboulos, and S. Vaidyanathan /

Journal of Engineering Science and Technology Review 8 (2) (2015) 157 - 173

\begin{tabular}{|cccc|}
\hline Name & Law & Constant $\boldsymbol{~ I t s ~ n a m e ~}$ \\
\hline Resistor & $i=\alpha v$ & $\alpha=1 / R$ & $R$ : Resistance \\
Capacitor & $\int i=\alpha v$ & $\alpha=C$ & $C$ : Capacitance \\
Inductor & $i=\alpha \int_{v}$ & $\alpha=1 / L$ & $L:$ Inductance \\
Memristor & $\int \mathrm{i}=\alpha \int_{v}$ & $\alpha=1 / M$ & $M:$ Memristance \\
\hline
\end{tabular}

Tab. 1. The possible relationships between current $(i)$ and voltage $(v)$.

So, until recently, the memristor had received little attention [3-5], even though a working device made from op-amps and discrete nonlinear resistors had been built and demonstrated in the seminal paper of Chua [1]. The memristor, we can say that it was the 'Holy Grail' of Electronics. However, in 2008, Hewlett-Packard scientists, working at its laboratories in Palo Alto-California, announced in Nature [6] that a physical model of memristor has been realized. In their scheme, a memory effect is achieved in solid-state thin film two-terminal device.

This element is passive while the latest realization of a memristor is that of an active one on a base of niobium oxide [7]. The memristor, which is realized by HP researchers, is made of a titanium dioxide layer which is located between two platinum electrodes. This layer is of the dimension of several nanometers and if an oxygen dis-bonding occurs, its conductance will rise instantaneously. However, without doping, the layer behaves as an isolator. The area of oxygen dis-bonding is referred to as space-charge region and changes its dimension if an electrical field is applied. This is done by a drift of the charge carriers. The smaller the insulating layer, the higher the conductance of the memristor. Also, the tunnel effect plays a crucial role. Without an external influence the extension of the space-charge region does not change.

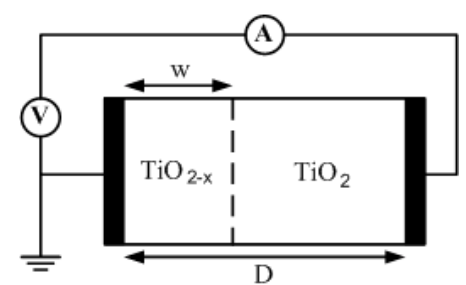

(a)

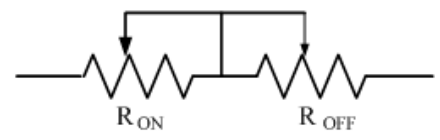

(b)

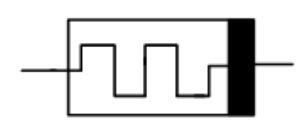

(c)

Fig. 2. Structure of $\mathrm{TiO}_{2}$ memristor, in which $\mathrm{TiO}_{2-\mathrm{x}}$ and $\mathrm{TiO}_{2}$ layers are sandwiched between two platinum electrodes, (b) equivalent circuit and (c) symbol of the memristor.

The internal state $x$ is the extent of the space-charge region, which is restricted in the interval $[0,1]$ and can be described by the equation
$x=\frac{w}{D}, \quad 0 \leq x \leq 1, \quad x \in \mathrm{R}$

where $w$ is the absolute extent of the space-charge region and $D$ is the absolute extent of the titanium dioxide layer. The memristance can be described by the following equation:

$$
M(x)=R_{\mathrm{on}} x+R_{\mathrm{off}}(1-x)
$$

where $R_{\text {on }}$ is the resistance of the maximum conducting state and $R_{\text {off }}$ represents the opposite case. So, when $x=0$, $R=R_{\text {off }}$, and when $x=1, R=R_{\text {on }}$. The vector containing the internal states of the memristor is one dimensional. For this reason scalar notation is used. The state equation is:

$\frac{\mathrm{d} x}{\mathrm{~d} t}=\frac{\mu_{v} R_{\mathrm{on}}}{D^{2}} i(t)$

where $\mu_{v}$ is the oxygen vacancy mobility and $i(t)$ is the current through the device. By using the equation (6) the previous equation can be rewritten as:

$\frac{\mathrm{d} w}{\mathrm{~d} t}=\frac{\mu_{v} R_{\mathrm{on}}}{D} i(t)$

So, the dynamics of the memristor can therefore be modeled through the time dependence of the width $w$ of the doped region. Integrating equation (9) with respect to time,

$w=w_{0}+\frac{\mu_{v} R_{\mathrm{on}}}{D} q(t)$

where $w_{0}$ is the initial width of the doped region at $t=0$ and $q$ is the amount of charges that have passed through the device. Substituting (6), (10) into equation (7) gives:

$M(q)=R_{0}-\frac{\mu_{v} R_{\mathrm{oo}} \Delta R}{D^{2}} q(t)$

where

$$
R_{0}=R_{\text {on }} \frac{w_{0}}{D}+R_{\text {off }}\left(1-\frac{w_{0}}{D}\right)
$$

and $\Delta R=R_{\text {off }}-R_{\text {on }}$. The term $R_{0}$ refers to the net resistance at $t=0$ that serves as the device's memory. This term is associated with the memristive state, which is essentially established through a collective contribution, i.e. it depends directly on the amount of all charges that have flown through the device. 
That's why, we can say that the memristor has the feature to 'remember' whether it is on or off after its power is turned on and off. This announcement brought a revolution in various scientific fields, as many phenomena in systems, such as in thermistors whose internal state depends on the temperature [8], spintronic devices whose resistance varies according to their spin polarization [9] and molecules whose resistance changes according to their atomic configuration [10], could be explained now with the use of the memristor. Also, electronic circuits with memory circuit elements could simulate processes typical of biological systems, such as the learning and associative memory [11] and the adaptive behavior of unicellular organisms [12].

Furthermore, neuromorphic computing circuits, which are designed by borrowing principles of operation typical of the human (or animal) brain, can potentially solve problems that are cumbersome (or outright intractable) by digital computation. Therefore, certain realizations of memristors can be very useful in such circuits because of their intrinsic properties which mimic to some extent the behavior of biological synapses. Just like a synapse, which is essentially a programmable wire used to connect groups of neurons together, the memristor changes its resistance in varying levels. Many research teams [13-16] found that memristors can simulate synapses because electrical synaptic connections between two neurons can seemingly strengthen or weaken depending on when the neurons fire. For this reason, many well-known circuits, in which the nonlinear element has been replaced by memristors, have been proposed [17-19].

Based on the aforementioned fact that memristors can mimic the behavior of biological synapses, we have studied, via computer simulations the dynamical behavior of two systems of coupled nonlinear neuromorphic circuits that simulates the well-known Hindmarsh-Rose and FitzHughNagumo neuron models. For this reason a flux-controlled memristor, as an electrical synapse, is used in each case. The proposed coupled systems have very interesting dynamic behavior as for certain values of their elements, they exhibit spiking/bursting and chaos phenomena, as it occurs in real neurons.

According to the related explanations, the rest of the chapter is organized as follows: The next Section provides a brief description of the most interesting applications of memristors in various scientific fields. Section 3 presents the scientific field of neuromorphic circuits, which is probably the most exciting application of memristors, while Section 4 presents the most well-known neuron models and the basic features of the synapse. In Section 5 the explanation of the relation between the synapse and the memristor is discussed. Section 6 is devoted to the results of the coupling schemes between the circuits that simulate the chosen neuron models via a memristor, which plays the role of a synapse. Finally, Section 6 outlines the conclusions that have been reached with this research study.

\section{Applications of Memristor}

What implications does the discovery of memristor hold for the future of electronics technology? If it is such an important circuit element, why has it taken nearly four decades to construct a prototype of the memristor? It is a fact that in history, we have several such instances of practical realizations lagging behind the theoretical conceptualization.
Also, as it is mentioned, due to its dynamical behavior, the memristor enables a lot of new interesting applications in analog circuit design. Since, some realizations have been already presented, the development of applications with memristors becomes more and more challenging. Besides applications in neural networks and storage devices, analog memristive circuits also promise further applications. In this section, some of the more interesting and promising applications of the memristors are presented in detail. This list of applications reveals that the research on the memristor is a subject that will be insisted on it in the near future.

- Digital Memory: Until now the most straightforward and developed application of memristive systems is the digital binary non-volatile memory. This occurs because a bit of information could be easily encoded in the memristive system's state assigning, for example, the low resistance state to ' 1 ' and the high resistance state to ' 0 '. For that reason many research teams have already work on this subject [20-22]. So, the use of memristor could offer an enabling low cost technology for non-volatile memories where future computers would turn on instantly without the usual 'booting time', currently required in all personal computers [17].

- Programmable Analog Circuits: In this circuits' family various memristive systems that operate under threshold conditions could be used as digital potentiometers, by applying small amplitude voltages to these systems when they are used as analog circuit elements [23]. In the case of memristive systems the state is changed only when the voltage applied to it exceeds a certain threshold. So, its resistance is constant in the analog mode of operation, and changes by discrete values with each voltage pulse.

- Learning Circuits: An electronic circuit whose response at a given time adapts according to signals applied to this at previous moments of time, is called 'learning circuit' [12]. This actually is the reason for which memory circuit elements would be used because they could provide non-volatile information storage. Such electronic circuits have already been proposed and experimentally implemented. Pershin et al. [12] proposed a learning circuit which is composed of an LC contour and a memristive system in parallel with a capacitor that mimics the adaptive behavior of a slime mold 'Physarum polycephalum' from the group of amoebozoa. Additionally, Driscoll et al. [24] realized a similar learning in which the application of signals in a specific frequency range sharpens the quality factor of its resonant response, and thus the circuit learns according to the input waveform.

- Quantum Computing with Memory Circuit Elements: Nowadays, the research topic of quantum computing becomes more and more interesting due to the fact that the usual computer technology reaches its limits. The superconducting qubit circuits, which are designed, involve usual capacitors and inductors [25, 26]. So, many research teams thought that the use of memory elements such as memcapacitive and meminductive are ideal for such circuits, especially for use in the fieldprogrammable quantum computing [27]. This would be done by replacing the capacitors and the inductors, which provide the coupling between different qubits, with non-dissipative memcapacitors and meminductors. So, by providing additional voltage sources to control the state of these memory elements the coupling 
strength between qubits can be selected. This would be beneficial from such novel quantum hardware design because of the infinity interaction schemes that can be implemented within a single circuit architecture.

- Digital Logic: One of the most promising applications of memristive systems could be their use to perform logic operations. There have already been demonstrated very promising hybrid reconfigurable logic circuits [28], and logic circuits with a 'self-programming' capability [29]. The state of the memristive system acts as both logic gate and latch capable to hold one bit of information. Lehtonen et al. [30] have reached to the interesting conclusion that two memristive systems suffice to compute all Boolean functions.

- Metamaterials: In 2009 Driscoll et al. demonstrated a form of memory capacitance that interfaces metamaterials with a class of devices known collectively as memory devices [31]. The resonant elements that grant metamaterials their distinct properties have the fundamental limitation of restricting their useable frequency bandwidth. The development of frequency-agile metamaterials has helped to alleviate these bandwidth restrictions by allowing real-time tuning of the metamaterial frequency response. So, Driscoll et al. demonstrated electrically controlled persistent frequency tuning of a metamaterial, which allows the lasting modification of its response by using a transient stimulus.

- Dynamic Load: Shin et al. have presented in 2010 a programmable gain amplifier that utilises a memristor as a variable resistor [32]. This implementation has the advantages of the increased step resolution of the effective resistance and the fact that this system is susceptible to minimal parasitics. Also, Pershin and Di Ventra have reported a similar approach in which they used memristors to develop programmable threshold comparators, Schmitt triggers and frequency relaxation oscillators [23].

- Image Processing: Memristive systems could be used for the edge detection which is an important computational step in early vision systems that finds application in various domains from computer vision to bio-imaging. This process is performed using either resource intensive software algorithms or by employing resistive grids, implemented with conventional CMOS elements. Prodromakis et al. (2010) have proposed the substitution of the CMOS elements with memristive elements serve as dynamic sensors which change in the total memristance value is a direct indication of the pixel intensity gradient between neighbouring pixels [33]. Since an edge is defined by a large intensity difference, the devices are monitored continuously and appropriate memristance thresholds are set to enable the faster or even slower detection of the edges, depending on the average pixel intensity contrast of the figure.

- Cellular Neural Networks: As it is known, the Cellular Neural Networks (CNN) constitute a class of information processing systems, which are made of massive aggregates of regularly spaced circuit clones, called cells that communicate with each other only through their nearest neighbors. In a memristive implementation of a $\mathrm{CNN}$ processor, the intercellular connections are implemented by memristive crossbars [18]. The motivation for using memristive crossbars is that the area consuming intercellular communication network can be lifted from the CMOS layer, thus allowing for a larger number of cells within the same die area.

- Other Applications: In the last few years new research results have appeared concerning the generation and analysis of chaotic signals using memristor elements. All these new techniques are based on the replacement of the nonlinear resistance in the circuit, like the wellknown Chua circuit [34], which is the most basic chaos generating circuit, by the memristor and novel features in chaotic behavior are observed. These attempts had as a motivation the use of such circuits for the purpose of modeling of dynamics which are shown in the nature. From this perspective, Itoh and Chua derived several oscillators from Chua's oscillators by replacing Chua's diodes with memristors characterized by a monotoneincreasing piecewise-linear function [35]. In the same way Muthuswamy and Kokate proposed other memristor based chaotic circuits [18]. Also, in 2010, Muthuswamy and Chua proposed an autonomous circuit that uses only three circuit elements in series: a linear passive inductor, a linear passive capacitor and a memristor [36]. Furthermore, in Refs. [19, 37-40] cubic memristors have replaced the nonlinear elements in well known circuits of Chua's family.

Apart from the interesting fundamental study of the dynamic behavior of nonlinear systems, the field of applications of such circuits also includes secure communication schemes with chaos based on memristors [18], the image stabilization [41] and image encryption technique by using memristors [42].

\section{Neuromorphic Circuits}

However the most exciting application of memristive systems and possibly, the most important is in neuromorphic circuits. Neuromorphic are circuits which operation is meant to mimic that of the (human or animal) brain. In these circuits, memristive systems (and possibly also memcapacitive systems) can be used as synapses whose role is to provide connections between neurons and store information. The small size of solid-state memristive systems is highly beneficial for this application since the density of memristive systems in a chip can be of the same order of magnitude as the density of synapses in human brains (1010 synapses per square centimeter). Therefore, using memristive systems, the fabrication of an artificial neural network of a size comparable to that of a biological brain becomes possible.

As it is known, a neuron, which is also known as 'neurone' or 'nerve cell', is an electrically excitable cell that processes and transmits information through electrical and chemical signals. A signal occurs via a synapse, a specialized connection with other cells. So, neurons connect to each other to form neural networks. Neurons are the core components of the nervous system, which includes the brain, spinal cord, and peripheral ganglia. A number of specialized types of neurons exist, such as [43]:

- Sensory neurons which respond to touch, sound, light and numerous other stimuli affecting cells of the sensory organs that then send signals to the spinal cord and brain.

- Motor neurons which receive signals from the brain and spinal cord, cause muscle contractions, and affect glands. 
- Interneurons which connect neurons to other neurons within the same region of the brain or spinal cord.

All neurons are electrically excitable, maintaining voltage gradients across their membranes by means of metabolically driven ion pumps, which combine with ion channels embedded in the membrane to generate intracellular-versus-extracellular concentration differences of ions such as sodium, potassium, chloride, and calcium. Changes in the cross-membrane voltage can alter the function of voltage-dependent ion channels. If the voltage changes by a large enough amount, an all-or-none electrochemical pulse called an action potential is generated, which travels rapidly along the cell's axon, and activates synaptic connections with other cells when it arrives.

Real neurons show a variety of dynamical behaviors, according to the values of biophysical parameters [44]. Among the most important ones, one may find:

- Quiescence: The input to the neuron is below a certain threshold and the output reaches a stationary regime.

- Spiking: The output is made up of a regular series of equally spaced spikes.

- Irregular Spiking: The output is made up of an aperiodic series of spikes.

- Bursting: The output is made up of groups of two or more spikes (called bursts) separated by periods of inactivity.

- Irregular Bursting: The output is made up of an aperiodic series of bursts.

The other important part in the nervous system is the synapse. The word 'synapse' was introduced in 1897 by English physiologist Michael Foster. Synapses are essential to neuronal function because they are the means by which the signals are passed from one neuron to another. At a synapse, the plasma membrane of the signal-passing neuron (the presynaptic neuron) comes into close apposition with the membrane of the target (postsynaptic) cell. Both the presynaptic and postsynaptic sites contain extensive arrays of molecular machinery that link the two membranes together and carry out the signaling process. [45]:

There are two fundamentally different types of synapses

- Chemical synapses: In this type of synapses, the electrical activity in the presynaptic neuron is converted (via the activation of voltage-gated calcium channels) into the release of a chemical called a neurotransmitter that binds to receptors located in the postsynaptic cell, usually embedded in the plasma membrane. The neurotransmitter may initiate an electrical response or a secondary messenger pathway that may either excite or inhibit the postsynaptic neuron. Because of the complexity of receptor signal transduction, chemical synapses can have complex effects on the postsynaptic cell.

- Electrical synapses: In this type of synapses, the presynaptic and postsynaptic cell membranes are connected by special channels called gap junctions that are capable of passing electric current, causing voltage changes in the presynaptic cell to induce voltage changes in the postsynaptic cell. The main advantage of an electrical synapse is the rapid transfer of signals from one neuron to the next.

\section{Neuron Models}

Neurons are a core component of the nervous system. Organized internally similar to other cells, they are specialized for intercellular communication by way of their membrane potential. Biological experiments and numerical analysis of models for the oscillations of isolated neurons, have led the researchers to construct low dimensional analog electronic neurons whose properties are designed to emulate the membrane voltage characteristics of the individual neurons. So, in the case of modeling a biological neuron, physical analogues are used in place of abstractions such as 'weight' and 'transfer function'. The input to a neuron is often described by an ion current through the cell membrane that occurs when neurotransmitters cause an activation of ion channels in the cell. We describe this by a physical timedependent current $I(t)$. The cell itself is bound by an insulating cell membrane with a concentration of charged ions on either side that determines a capacitance $C_{m}$. Finally, a neuron responds to such a signal with a change in voltage, or an electrical potential energy difference between the cell and its surroundings, which is observed to sometimes result in a voltage spike called an action potential. This quantity, then, is the quantity of interest and is given by $V_{m}$. Until now, many models of biological neurons have been reported in the literature. Next, the most important models, which will be used in the next Section, are presented in detail.

\subsection{Hodgkin-Huxley model}

It is the most successful and widely-used model of neuron, which has been based on the Markov kinetic model developed from Hodgkin and Huxley's 1952 work based on data from the squid giant axon [46]. This model tries to replicate the electrophysiological process of biological neurons.

In more details, the semi-permeable cell membrane separates the interior of the cell from the extracellular liquid and acts as a capacitor (Fig.3). If an input current $I(t)$ is injected into the cell, it may add further charge on the capacitor, or leak through the channels in the cell membrane. Because of active ion transport through the cell membrane, the ion concentration inside the cell is different from that in the extra-cellular liquid. The 'Nernst' potential generated by the difference in ion concentration is represented by a battery. The conservation of electric charge on a piece of membrane implies that the applied current $I(t)$ may be split in a capacitive current $I_{C}$ which charges the capacitor $C$ and further components $I_{k}$ which pass through the ion channels. Thus

$$
I(t)=I_{C}(t)+\sum_{k} I_{k}(t)
$$

where the sum runs over all ion channels. In the standard Hodgkin-Huxley model there are only three types of channel: a sodium channel with index $N a$, a potassium channel with index $K$ and an unspecific leakage channel with resistance $R$ (Fig.3).

From the definition of capacity $C=Q / u$ where $Q$ is a charge and $u$ the voltage across the capacitor, we find the charging current $I_{C}=C \mathrm{~d} u / \mathrm{d} t$. Hence from (13): 
$C \frac{\mathrm{d} u}{\mathrm{~d} t}=I(t)-\sum_{k} I_{k}(t)$

In biological terms, $u$ is the voltage across the membrane and $\sum_{k} I_{k}(t)$ is the sum of the ionic currents which pass through the cell membrane.

\section{Outside}
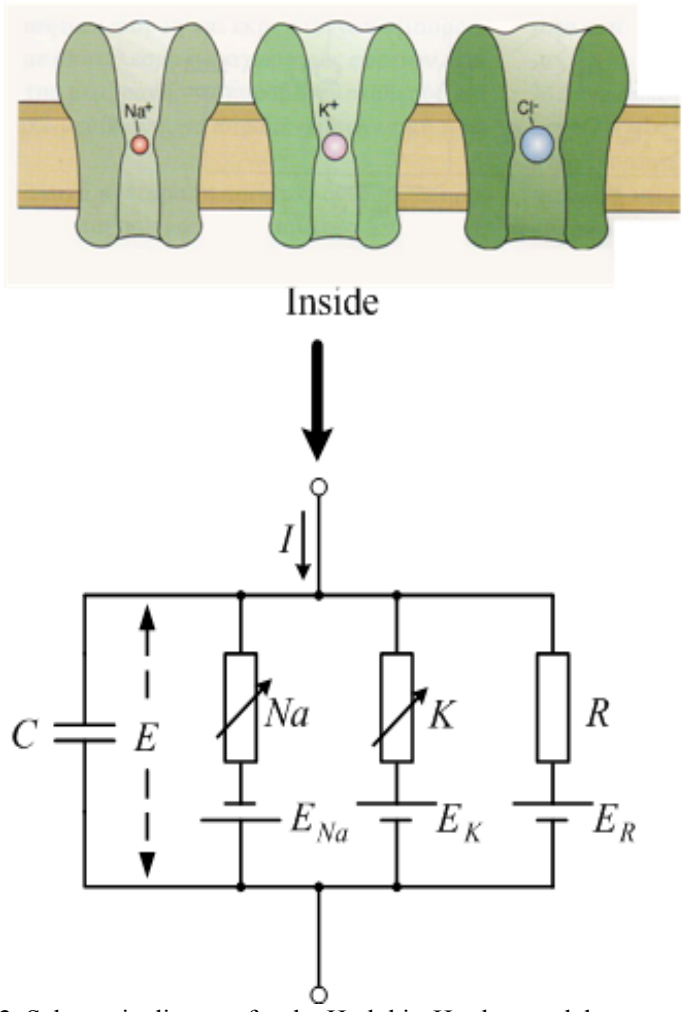

Fig. 3. Schematic diagram for the Hodgkin-Huxley model.

As mentioned above, the Hodgkin-Huxley model describes three types of channel. All channels may be characterized by their resistance or, equivalently, by their conductance. The leakage channel is described by a voltageindependent conductance $g_{L}=1 / R$; the conductance of the other ion channels is voltage and time dependent. If all channels are open, they transmit currents with a maximum conductance $g_{N a}$ or $g_{K}$, respectively. Normally, however, some of the channels are blocked. The probability that a channel is open is described by additional variables $m, n$ and $h$. The combined action of $m$ and $h$ controls the $\mathrm{Na}^{+}$ channels. The $K^{+}$gates are controlled by n. Specifically, Hodgkin and Huxley formulated the three current components as:

$$
\sum_{k} I_{k}=g_{N a} m^{3} h\left(u-E_{N a}\right)+g_{K} n^{4}\left(u-E_{K}\right)+g_{L}\left(u-E_{L}\right)
$$

where the parameters $E_{N a}, E_{K}$, and $E_{L}$ are the reversal potentials.

The three variables $m, n$, and $h$ are called gating variables. They evolve according to the differential equations

$$
\left\{\begin{array}{l}
\frac{\mathrm{d} m}{\mathrm{~d} t}=\alpha_{m}(u)(1-m)-\beta_{m}(u) m \\
\frac{\mathrm{d} n}{\mathrm{~d} t}=\alpha_{n}(u)(1-n)-\beta_{n}(u) n \\
\frac{\mathrm{d} h}{\mathrm{~d} t}=\alpha_{h}(u)(1-h)-\beta_{h}(u) h
\end{array}\right.
$$

where $\alpha$ and $\beta$ are empirical functions of $u$ that have been adjusted by Hodgkin and Huxley to fit the data of the giant axon of the squid.

\subsection{FitzHugh-Nagumo model}

Sweeping simplifications to Hodgkin-Huxley were introduced by FitzHugh and Nagumo [47]. Seeking to describe 'regenerative self-excitation' by a nonlinear positive-feedback membrane voltage and recovery by a linear negative-feedback gate voltage, they developed the model described by

$$
\left\{\begin{array}{l}
\frac{\mathrm{d} x}{\mathrm{~d} t}=\gamma\left(x-\frac{1}{3} x^{3}+y+z\right) \\
\frac{\mathrm{d} y}{\mathrm{~d} t}=-\frac{1}{\gamma}(x-\alpha+\beta y)
\end{array}\right.
$$

where the variable $x$ describes the potential difference across the neural membrane and $y$ can be considered as a combination of the different ion channel conductivities, present in the Hodgkin-Huxley model. The control parameter $z$ of the FitzHugh system describes the intensity of the stimulating current.

\subsection{Hindmarsh-Rose model}

The Hindmarsh-Rose (HR) model is based on the global behavior of the neuron and its underlying operation is removed from the actual biological process. For this reason, is one of the most interesting neuron models which is used for studying the neuronal activity and more specifically the spiking-bursting behavior of the membrane potential observed in experiments made with a single neuron. This phenomenological neuron model, which has been proposed by Hindmarsh and Rose [48], may be seen either as a generalization of the Fitzhugh equations or as a simplification of the physiologically realistic model proposed by Hodgkin and Huxley. It has been proven to be a single-compartment model providing a good compromise between two seemingly mutually exclusive requirements: The model for a single neuron must be both computationally simple, and capable of mimicking almost all the behaviors exhibited by real biological neurons, in particular the rich firing patterns [49].

So, the three-variable HR model of action potential was proposed as a mathematical representation of the firing behavior of neurons, and it was originally introduced to give a bursting type with long InterSpike Intervals (ISIs) of real neurons. It can be used to simulate spiking/bursting and chaos phenomena in real neurons. The equations of the HR model are given as follows: 


$$
\left\{\begin{array}{l}
\frac{\mathrm{d} X}{\mathrm{~d} \tau}=Y-a \cdot X^{3}+b \cdot X^{2}-Z+I \\
\frac{\mathrm{d} Y}{\mathrm{~d} \tau}=c-d \cdot X^{2}-Y \\
\frac{\mathrm{d} Z}{\mathrm{~d} \tau}=r \cdot(s \cdot(X+\chi)-Z)
\end{array}\right.
$$

where, $X$ represents the membrane action potential, $Y$ is a recovery variable and $Z$ is a slow adaptation current; $I$ mimics the membrane input current for the biological neurons; $a, b$ allows one to switch between bursting and spiking behaviors and to control the spiking frequency; $r$ controls the speed of variation of the slow variable $Z$ in (18), (i.e., the efficiency of the slow channels in exchanging ions) and in the presence of spiking behaviors, it governs the spiking frequency, whereas in the case of bursting, it affects the number of spikes per burst; $s$ governs adaptation: $a$ unitary value of $s$ determines spiking behavior without accommodation and sub-threshold adaptation, whereas values around $s=4$ give strong accommodation and sub-threshold overshoot, or even oscillations; $\chi$ sets the resting potential of the system.

In the following numerical simulations, let $a=1.00$, $b=2.82, c=1.00, d=5.00, \mathrm{r}=0.02, s=4.00, \chi=-1.60$ and $I=3.50$. As it can be shown in Figure 4(a), the HR model shows the typical bursting neuronal behavior, in which spikes are separated by periods of inactivity, while in Figure 4(b) the chaotic behavior of the model for the selected parameter's values is confirmed. Each semi-circle originates from an individual spike from the overall output burst.

Next, the FitzHugh-Nagumo and Hindmarsh-Rose neuron models will be the subject of study by using coupling schemes in which a memristor has been used as an artificial synapse.

\section{The Use of Memristor as a Synapse}

Communication and coordination between neurons is made possible by synapses. When a synapse connects two neurons together the 'postsynaptic' neuron receives the ionic current while the 'presynaptic' neuron is the source. Also, in living nervous systems one finds three general types of synaptic connections among neurons [50]: ohmic electrical connections (also called gap junctions), which is usually found in the nervous system of all animals, and two types of chemical connections, excitatory and inhibitory, which are the more common. So, a synapse is essentially a programmable wire used to connect together groups of neurons.

Neuromorphic computers which aim at mimicking biological computation, and have numbers of neurons and synapses approaching biological scale, can be modeled with supercomputers or neural hardware accelerators. However, in order for such neural computing devices to achieve a biologically plausible synaptic density, it is imperative to minimize synaptic size.

This feat is challenging because the synaptic weight of each synapse must be stored. Since digital synapse implementations require that several bits of data per synapse are memorized, analog synapses may be a superior choice. Analog synapses based on floating-gate transistors store the weight as charge that is trapped between insulating layers. The charge can be manipulated by injecting and tunnelling electrons to and from the floating node. Such transistors rely on proven technology and allow a relatively high density, rendering them worthwhile synaptic candidates. However, the memristors, this new class of devices, is the next leap forward to high density synapse fabrication. Memristive devices will allow the fabrication of single device synapses as crossbar arrays on top of Complimentary Metal-OxideSemiconductor (CMOS) circuits. As the synapses would be in the memristive layer on top of the CMOS, the entire silicon area would be left for neurons.
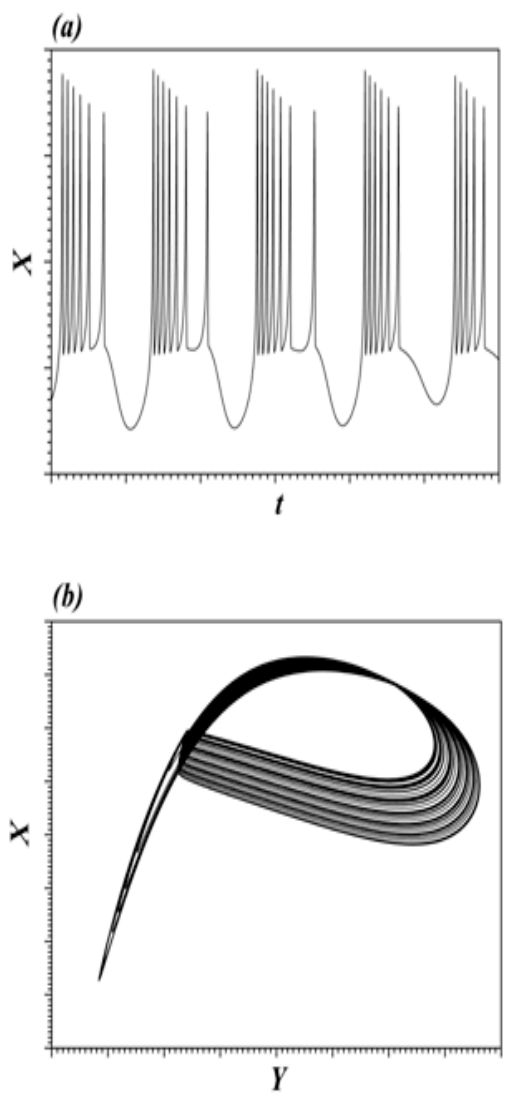

Fig. 4. Simulation of Hindmarsh-Rose neuron showing (a) a typical chaotic bursting neuronal behavior for the membrane potential $(X)$ and (b) a chaotic phase portrait of $Y$ vs. $X$.

Some of the characteristics of a memristor synapse in comparison with various other CMOS designs, like low power consumption (because of nonvolatile capacitor-like weight memory and less transistor counts), linear behavior of the network (because of linear multiplier), more speed in the operation phase (because of using very fast memristor multiplier instead of slow Gilbert multipliers) and smaller size (because of the replacement of many transistors and a big capacitor with a nanometer memristor), make the memristors suitable for use in artificial neural networks.

Until now, various memristors and techniques are used for studying their behavior as an artificial synapse. Afifi et al. (2009) have proposed and analyzed Spike-TimingDependent-Plasticity (STDP) rule for memristor crossbar based spiking neuromorphic networks [51]. Sharifi et al. (2010) by using the characteristics, structures and relations for the invented HP's memristor, designed two general SPICE models for the charge-controlled and flux-controlled 
memristors, which are used as synapses in an artificial neural network [52]. A pulse-based programmable memristor circuit for implementing synaptic weights for artificial neural networks is proposed by Kim et al. [53]. Also, in 2012, Sah et al. have implemented a memristor bridge neural circuit, by using memristor emulator circuits, which is able to perform signed synaptic weighting [54].

In 2010, piecewise linear $\varphi-q$ characteristics of a flux controlled memristor [17], or characteristics with other forms of smooth continuous nonlinearities [55], have been proposed for using in nonlinear circuits design. Also, other researchers have proposed memristors having cubic nonlinearities $[18,19,35,56]$ of the form $q(\varphi)=\alpha \varphi+\beta \varphi^{3}$, with $\alpha<0$ and $\beta>0$. Snider (2008) and Linares-Barranco (2009) have proposed memristors with sinh-like characteristic curves, which have been used to explain spiketime-dependent-plasticity in neural synapses $[16,57]$.

In this direction as an approximation of the aforementioned sinh-like characteristic curves, for studying the interconnections among coupled analog electronic neurons a flux-controlled memristor is used, to emulate the excitatory and inhibitory synaptic connection as well as the ohmic electrical connections. The proposed memristor is described by the function $w(\varphi)$, where $q(\varphi)$ is a smooth continuous cubic function of the form:

$$
q(\varphi)=\mathrm{k}_{1} \varphi+\mathrm{k}_{3} \varphi^{3}
$$

with $k_{1}, k_{3}>0$. As a result the memductance $w(\varphi)$ is provided by the following expression:

$$
w(\varphi)=\frac{\mathrm{d} q(\varphi)}{\mathrm{d} \varphi}=\mathrm{k}_{1}+3 \mathrm{k}_{3} \varphi^{2}
$$

So, it will be shown that the proposed memristor acts as an artificial synapse between coupled neuron-cells.

\section{Coupled Neuromorphic Circuits}

In this Section two different circuits, which realized the Hindmarsh-Rose and the FitzHugh-Nagumo neuron models, are coupled via the previously mentioned memristor that is used as an artificial synapse. The proposed systems are solved numerically by applying the fourth-order RungeKutta algorithm and various tools of nonlinear dynamics such as the bifurcation diagram and the phase portraits have been used. As it will be presented in detail, the coupled neuronal systems show interesting dynamical behavior, such as chaos, periodic behavior and synchronization.

Systems of chaotic oscillators, which are coupled, are frequently found not only in the simulation environment or the laboratory but also in the natural world. For this reason, many techniques for coupling two or more nonlinear chaotic systems have been proposed in the literature. All these techniques can be mainly divided into two classes: 'unidirectional coupling' and 'bidirectional or mutual coupling'. In the unidirectional coupling, one system drives another one, while on the contrary in mutual coupling both the circuits are connected and each circuit's behavior influences the dynamics of the other. The case of mutual coupling between two coupled chaotic oscillators is described by the following set of differential equations:

$$
\left\{\begin{array}{l}
\dot{x}_{1}=F\left(x_{1}\right)-C \cdot\left(x_{1}-x_{2}\right) \\
\dot{x}_{2}=G\left(x_{2}\right)+C \cdot\left(x_{1}-x_{2}\right)
\end{array}\right.
$$

while in the case of unidirectional coupling the system of differential equation is written as:

$$
\left\{\begin{array}{l}
\dot{x}_{1}=F\left(x_{1}\right) \\
\dot{x}_{2}=G\left(x_{2}\right)+C \cdot\left(x_{1}-x_{2}\right)
\end{array}\right.
$$

where $F(x), G(x)$ are vector fields in the phase space of dimension $n$, i.e. $x \in \mathrm{R}^{n}$, and $C$ is a symmetric matrix of constants which describes the nature and strength of the coupling between the oscillators.

Electrical synapses are usually bidirectional but in some cases can operate heavier in one direction than the other, or only in one direction [50]. They are created when the presynaptic and postsynaptic membranes meet and gap junction channels of each align. Ions flow through gap junctions proportionally to the potential differential across them. So, electrical synapses are quick message carriers.

On the other hand, chemical synapses are more commonly found in biology. Unlike the electrical synapse, there is no direct connection between the presynaptic and postsynaptic neurons. No ionic current flows between neurons, but neurotransmitters secreted by the presynaptic neuron open receptors for special molecules [58]. These molecules which are allowed to flow through the receptors have a postsynaptic potential response which either increases (excitatory coupling) or decreases (inhibitory coupling) the membrane potential.

The basic tool of the study of the dynamic behavior of the coupled neuron models is the well-known 'bifurcation diagram', which is a very common perspective in nonlinear dynamics. Also, in order to study how a system depends on the initial values of the state variables, two different bifurcation diagrams are produced numerically. In the first approach, the bifurcation diagram is produced by increasing the coupling factor $\xi$, from $\xi=0$ (uncoupled system) to $\xi_{\max }$ with step $\Delta \xi$, while initial conditions in each iteration have different values. This occurs because the last values of the state variables in the previous iteration become the initial values for the next iteration. In the second approach, the bifurcation diagram is produced by using the same initial conditions in each iteration. This means that the system begins, in each iteration, for the same basin of attraction.

Finally, the most interesting phenomenon and one of the objectives of this work is the study of the synchronization of the coupled neuron-cells. Synchronization is a phenomenon characteristic of many processes in natural systems and especially in non-linear science [59]. It has remained an objective of intensive research and today it is considered as one of the basic nonlinear phenomena studied in mathematics, physics, engineering or life science [60]. Synchronization of nonlinear oscillators is also widely studied in biological systems [61, 62] for understanding how large and small neural assemblies efficiently and sensitively achieve desired functional goals [63].

In general, synchronization of chaos is a process, where two or more chaotic systems adjust a given property of their motion to a common behavior, such as equal trajectories or phase locking, due to coupling or forcing. 
So, in the case of 'full or complete chaotic synchronization', which is the most studied type of synchronization [64-67], two or more chaotic systems follow the same trajectory, i.e.,

$x_{1}(t)=x_{2}(t)$ as $t \rightarrow \infty$

\subsection{Coupled Hindmarsh-Rose Circuits}

The schematic of the circuit which makes the analog simulation of a Hindmarsh-Rose neuron model of equation (18) is shown in Fig.5(a). It consists of three integrators $\{1\}-$ $\{3\}$, two inverters $\{4\},\{5\}$ and two multipliers $\{6\},\{7\}$. The integrators and inverters can be implemented by using the operational amplifiers TL082 while the multipliers by using the AD633, which has high impedance differential inputs and a $10 \mathrm{~V}$ scaled output. The voltages of the positive and negative power supplies were set $\pm 15 \mathrm{~V}$.

The circuit of Fig.5(a) is described by the following set of differential equations.

$$
\left\{\begin{array}{l}
\frac{\mathrm{d} x}{\mathrm{~d} t}=\frac{1}{R C}\left[y-z+\frac{R}{10 R_{1}} x^{2}-\frac{R}{100 R_{2}} x^{3}+V_{1}\right] \\
\frac{\mathrm{d} y}{\mathrm{~d} t}=\frac{1}{R C}\left[V_{2}-\frac{R}{10 R_{3}} x^{2}-y\right] \\
\frac{\mathrm{d} z}{\mathrm{~d} t}=\frac{1}{R C}\left[\frac{R}{R_{4}}\left(x+V_{2}\right)-\frac{R}{R_{5}} z\right]
\end{array}\right.
$$

By introducing the new variables and parameters, $X=\frac{x}{V_{0}}$,

$\mathrm{Y}=\frac{y}{V_{0}}, \quad Z=\frac{z}{V_{0}} \quad$ and $\quad a=\frac{R}{100 R_{2}}, \quad b=\frac{R}{10 R_{1}}, \quad I=\frac{V_{1}}{V_{0}}$, $c=\frac{V_{2}}{V_{0}}, d=\frac{R}{10 R_{3}}, r=\frac{R}{R_{5}}, s=\frac{R_{5}}{R_{4}}, \chi=\frac{V_{2}}{V_{0}}$ and $\tau=\frac{1}{R C} t$, the normalized system of Hindmarsh-Rose neuron model of Eq.(18), for $a=1.0$ and $c=1.0$, is obtained. In Table 2 the values of circuit's elements in order to have the specific values of system's parameters, $b=2.82, d=5.0, r=0.02$, $s=4.0, \chi=1.6$ and $I=3.5$, are listed.

\begin{tabular}{|l|}
\hline$R=10.00 \mathrm{k} \Omega$ \\
\hline$R_{1}=354.60 \Omega$ \\
\hline$R_{2}=100.00 \Omega$ \\
\hline$R_{3}=200.00 \Omega$ \\
\hline$R_{4}=0.25 \mathrm{M} \Omega$ \\
\hline$R_{5}=0.50 \mathrm{M} \Omega$ \\
\hline$C=10.00 \mathrm{nF}$ \\
\hline$V_{0}=1.00 \mathrm{~V}$ \\
\hline$V_{1}=3.50 \mathrm{~V}$ \\
\hline$V_{2}=1.00 \mathrm{~V}$ \\
\hline
\end{tabular}

Tab. 2. The values of circuit's elements.

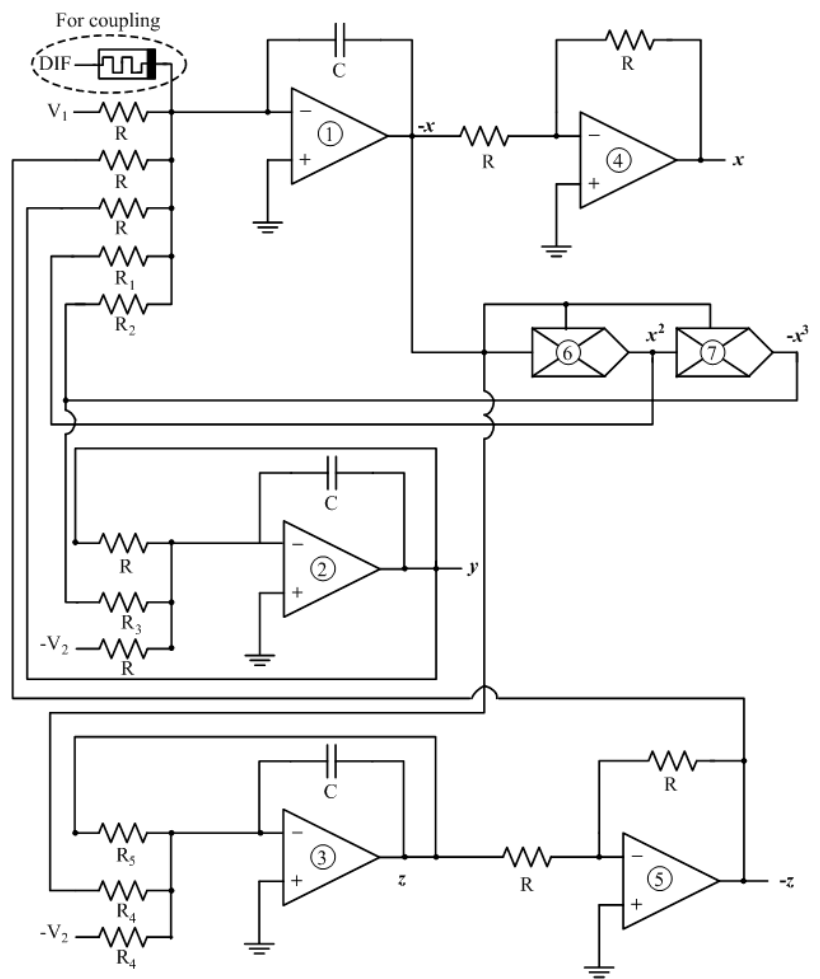

(a)

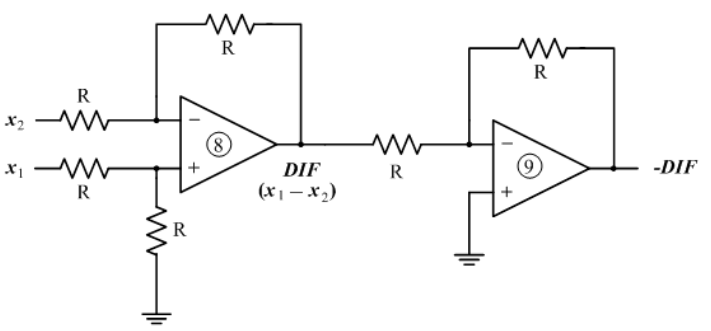

(b)

Fig. 5. (a) Schematic diagram of the Hindmarsh-Rose circuit; (b) Circuit realization for producing the difference signal $D I F=\left(x_{1}-x_{2}\right)$ and $-D I F$, which are used in the coupling scheme via the memristor.

Next, a system of two HR neuron model's circuits coupled via the proposed memristor, which acts as an artificial synapse, is studied. For this reason, the two different approaches of coupling (mutual and unidirectional) are used in order to study system's behavior.

In the first case, the network of two HR neuron model's circuits, electrically coupled via the proposed memristor is denoted as $N_{1} \leftrightarrow N_{2}$, in which $N_{1}$ and $N_{2}$ represent the two mutually coupled neurons, and the arrow denotes the electrical synaptic transfer direction. Also, as a stimulation a AC signal $\left(V_{1}=V_{\mathrm{A}} \cos (2 \pi f t)\right.$ is used in the first neuron.

For implementing the mutual type of coupling, between the HR circuits, a differential amplifier with op-amp $\{8\}$, which realize the signal $D I F=\left(x_{1}-x_{2}\right)$ is used (Fig.5(b)). Also, the inverter $\{9\}$ is used for inverting the signal $D I F$ in the case of the second coupled circuit. So, the first circuit models a HR neuron which is stimulated, while the second is a neuron which is coupled with the first one via the memristor that plays the role of an artificial synapse.

By applying the first Kirchhoff law, we obtain:

$$
\frac{V_{1}}{R}+\frac{1}{R} y-\frac{1}{R} z+\frac{1}{10 R_{1}} x^{2}-\frac{1}{100 R_{2}} x^{3}+i_{\mathrm{M}}=-C \frac{\mathrm{d} x}{\mathrm{~d} t}
$$


where,

$$
i_{M}=w(\varphi) v_{M}
$$

and $w(\varphi)$ the memductance is provided by the equation (22). So, by using the previously introduced normalized variables and the normalized variable of the memristor, $U=\varphi /\left(V_{0} R C\right)$, the coupled system can be described by the following normalized system of differential equations (29).

$$
\left\{\begin{array}{l}
\frac{\mathrm{d} X_{1}}{\mathrm{~d} \tau}=Y_{1}-X_{1}^{3}+b X_{1}^{2}-Z_{1}+I_{0} \cos \left(2 \pi f_{\mathrm{N}} \tau\right)- \\
\frac{-\left(\xi_{1}+3 \xi_{3} U^{2}\right)\left(X_{1}-X_{2}\right)}{\frac{\mathrm{d} Y_{1}}{\mathrm{~d} \tau}=c-d X_{1}^{2}-Y_{1}} \\
\frac{\mathrm{d} Z_{1}}{\mathrm{~d} \tau}=r\left[s\left(X_{1}+\chi\right)-Z_{1}\right] \\
\frac{\mathrm{d} X_{2}}{\mathrm{~d} \tau}=Y_{2}-X_{2}^{3}+b X_{2}^{3}-Z_{2}+\left(\xi_{1}+3 \xi_{3} U^{2}\right)\left(X_{1}-X_{2}\right) \\
\frac{\mathrm{d} Y_{2}}{\mathrm{~d} \tau}=c-d X_{2}^{2}-Y_{2} \\
\frac{\mathrm{d} Z_{2}}{\mathrm{~d} \tau}=r\left[s\left(X_{2}+\chi\right)-Z_{2}\right] \\
\frac{\mathrm{d} U}{\mathrm{~d} \tau}=X_{1}-X_{2}
\end{array}\right.
$$

where, $\xi_{1}=R k_{1}$ and $\xi_{3}=R^{3} V_{0}^{2} C^{2} k_{3}$.

The first three equations of system (29) represent the first HR stimulated neuron, the next three represent the second HR neuron and the last one is the proposed memristor's equation. For studying the effect of the proposed memristor to coupled system's behavior the normalized amplitude is $I_{0}=V_{\mathrm{A}} / V_{0}=3.5$ and the normalized frequency is chosen to be $f_{N}=R C f=0.16$, while the rest of the parameters remain the same.

In Fig.6(a) the bifurcation diagram (first approach) of system's (38) behavior, by plotting the signal difference $\left(Y_{2}-Y_{1}\right)$, with respect to the bifurcation parameter, is shown. At the beginning, the $\xi_{1}$ is chosen as a bifurcation parameter, while the other parameter of the memristor, the $\xi_{3}$ remains equal to zero. In this case, the two neuron-cells are linearly coupled, so the memristor simulates as a gap junction between them.

For extremely low values of the coupling factor $\xi_{1}$ the first neuron-cell shows the typical chaotic bursting neuronal behavior of Fig.4, under the influence of the stimulation, while the second neuron-cell is inactive. For greater values of $\xi_{1}$ (i.e., $\xi_{1}=0.02$ ) the coupled system is also in a chaotic state, as it is clearly shown from the phase portrait of $Y_{2}$ versus $Y_{1}$ (Fig.6(b)). However, for these values the second neuron-cell begins to oscillate chaotically as it is shown for the phase portrait of $Y_{2}$ versus $X_{2}$ in Fig.6(c). The range of this chaotic region is gradually narrowed as the coupling factor increases and ultimately results in a periodic state for $\xi_{1}>0.0226$. Fig.6(d) displays the system's period-1 attractor in the $Y_{2}$ versus $Y_{1}$ phase portrait.

In the second type of coupling (unidirectional coupling), the network of two HR neuron model's circuits are coupled again via the proposed memristor, but in this case only the second cell $N_{2}$ is influenced by the first one $N_{1}$, which is stimulated by the external AC signal $\left(N_{1} \rightarrow N_{2}\right)$. The coupled system, in the case of unidirectional coupling, can be described by the following normalized system of differential equations (30).

$$
\left\{\begin{array}{l}
\frac{\mathrm{d} X_{1}}{\mathrm{~d} \tau}=Y_{1}-X_{1}^{3}+b X_{1}^{2}-Z_{1}+I_{0} \cos \left(2 \pi f_{\mathrm{N}} \tau\right) \\
\frac{\mathrm{d} Y_{1}}{\mathrm{~d} \tau}=c-d X_{1}^{2}-Y_{1} \\
\frac{\mathrm{d} Z_{1}}{\mathrm{~d} \tau}=r\left[s\left(X_{1}+\chi\right)-Z_{1}\right] \\
\frac{\mathrm{d} X_{2}}{\mathrm{~d} \tau}=Y_{2}-X_{2}^{3}+b X_{2}^{3}-Z_{2}+\left(\xi_{1}+3 \xi_{3} U^{2}\right)\left(X_{1}-X_{2}\right) \\
\frac{\mathrm{d} Y_{2}}{\mathrm{~d} \tau}=c-d X_{2}^{2}-Y_{2} \\
\frac{\mathrm{d} Z_{2}}{\mathrm{~d} \tau}=r\left[s\left(X_{2}+\chi\right)-Z_{2}\right] \\
\frac{\mathrm{d} U}{\mathrm{~d} \tau}=X_{1}-X_{2}
\end{array}\right.
$$

So, in Figs.6(e) \& 6(f) the bifurcation diagrams in the two aforementioned approaches, with the same and different initial conditions in each step, are shown respectively. In general, these two diagrams has the same structure as the system begins in each case from the chaotic state $\left(\xi_{1}=0.02\right)$ and finally result in a periodic state (period-1), for $\xi_{1}>7.31$.

However, there is a great difference between them. In the first bifurcation diagram of Fig.6(e) the system passes through an intermediate chaotic region, while in the second one (Fig.6(f)) the system has a periodic behavior in the respective region, in which the period of the system is gradually decreased and finally results to a period-1. This difference is due to the well-known phenomenon of 'multistability' [68] in which the state of the coupled system, for a specific value of the coupling factor, may be different for the same set of system's parameters but for different initial conditions. Also, the coupling factor, for which the system results in a period- 1 state, is much greater than in the bidirectional coupling of Fig.6(a). This occurrs because in the bidirectional coupling the coupled neurons interact and the system goes to its final state for small values of $\xi_{1}$. In contrary, in the unidirectional coupling only the first neuroncell affects the dynamic behavior of the second neuron-cell, so greater values of the coupling factor is needed for achieving the final state.

Next, for studying the influence of the second term $\xi_{3}$ of the memristor, the parameter $\xi_{1}$ is chosen to be equal to 0.02 , so as the system is in chaotic mode according to the previous analysis, while $\xi_{3}$ varies. In the bidirectional coupling of neuron-cells, the memristor leads the system from the chaotic desynchronization for $\xi_{3}=0$ to an approximate synchronization mode. Also, a sudden transition from the irregular chaotic bursting behavior of each neuron, for $\xi_{3}=0$, to the periodic spiking behavior, for greater values of $\xi_{3}=0$, is observed. This is due to the way of coupling (mutually), which allows the interaction between the coupled cells by leading the system to a totally different dynamic behavior.

The synchronization of the mutually coupled neuroncells is quantified by calculating the difference $\Delta Y=Y_{1}-Y_{2}$ and studying the normalized standard deviation $\sigma_{N}=\Delta Y / Y_{1}$. For this reason a diagram of $\sigma_{N}$ versus the parameter $\xi_{3}$ has been made (Fig.6(g)). As it is shown, the deviation in the case of bidirectional coupling (black line) has decreased 
with the increasing of $\xi_{3}$ and finally for $\xi_{3}>0.0925$ the normalized standard deviation is smaller than $2 \%$. This means that the synchronization of the coupled neuron-cells is almost complete, as it shown in Fig.6(h). So, the memristor contributes to the synchronization of the coupled neuron-cells.

In the case of the unidirectionally coupled neuron-cells the use of the proposed memristor has the same effect as in the previous case. The coupled system is driven again from the chaotic desynchronization to the synchronization as the parameter is increased. But in this approach each one of the coupled neuron-cells is in a chaotic mode. So, a transition from the independent chaotic behavior to the synchronized chaotic bursting is observed. Also, the normalized standard deviation $\sigma_{N}$ (gray line in Fig.6(g)) is decreased with the increasing of the parameter $\xi_{3}$ but with smaller rate due to the coupling way. So, the system is driven again to a synchronization mode with a small deviation.

\subsection{Coupled FitzHugh-Nagumo Circuits}

As introduced by Fitzhugh (1961), the Bonhoeffer - van der Pol (BvP) model, for a spiking neuron, is a two dimensional reduction of the Hodgkin-Huxley equations [46]. A qualitative description of the single neuron activity is given, according to FitzHugh, by the system of coupled nonlinear differential equations (17). In 1962, Nagumo et al. [69] proposed an electronic simulator of the BvP model of FitzHugh using a tunnel diode as the nonlinear element (Fig.7(a)).

In this chapter a different approach for the analog simulation of the BvP model of differential equations (17), by using a nonlinear resistor with a smooth cubic $i-v$ characteristic, is adopted (Fig.7(b)). The smooth cubic $i$ - $v$ characteristic of the nonlinear resistor of this circuit is given by the following equation:

$$
i_{N}=g(v)=-\frac{1}{\rho}\left(v-\frac{1}{3} \frac{v^{3}}{V_{0}^{2}}\right)
$$

where $\rho$ and $V_{0}$ are normalization parameters.

From Kirchhoff's laws, the following equations are obtained:

$$
\left\{\begin{array}{l}
\frac{\mathrm{d} v}{\mathrm{~d} t}=\frac{1}{C}\left\{\frac{1}{\rho}\left(v-\frac{1}{3} \frac{v^{3}}{V_{0}^{2}}\right)+i_{L}+i_{S}\right\} \\
\frac{\mathrm{d} i_{L}}{\mathrm{~d} t}=\frac{1}{L}\left(-v-\mathrm{R} i_{L}+E\right)
\end{array}\right.
$$

By introducing new, normalized variables, $\tau=\frac{t}{\sqrt{L C}}$, $x=\frac{v}{V_{0}}, y=\frac{\rho i_{L}}{V_{0}}$ and $z=\frac{\rho i_{S}}{V_{0}}$, system (32) is reduced to differential equations of system (17), where, $\alpha=\frac{E}{V_{0}}$, $\beta=\frac{R}{\rho}$ and $\gamma=\frac{1}{\rho} \sqrt{\frac{L}{C}}$.
However, Rajasekar and Lakshmanan proposed a slightly different form of $\mathrm{BvP}$ oscillator $[70,71]$ given by the following state equations:

$$
\left\{\begin{array}{l}
\frac{\mathrm{d} x}{\mathrm{~d} \tau}=x-\frac{1}{3} x^{3}-y+z \\
\frac{\mathrm{d} y}{\mathrm{~d} \tau}=c(x+a-b y)
\end{array}\right.
$$

The study of system (33) revealed the existence of chaotic behavior, following the period doubling route to chaos, and devil's staircases. The nonlinear differential equations (33) can be also simulated by a nonlinear electric circuit, using a nonlinear resistor with the same smooth cubic $i-v$ characteristic as before (Fig.7(c)). By applying again the two Kirchhoff's laws we obtain the following equations.

$i_{C}+i_{L}+i_{N}=i_{S}$

and

$v=-E+R i_{L}+L \frac{\mathrm{d} i_{L}}{\mathrm{~d} t}$

By introducing the new, normalized variables, $\tau=\frac{t}{\rho C}$, $x=\frac{v}{V_{0}}, y=\frac{\rho i_{L}}{V_{0}}$ and $z=\frac{\rho i_{S}}{V_{0}}$, equations (34) and (35) are reduced to equations (33), where, $\alpha=\frac{E}{V_{0}}, \quad \beta=\frac{R}{\rho} \quad$ and $c=\frac{\rho^{2} C}{L}$.

In the aforementioned circuits of Figs.7(b) \& 7(c), the driving source is a current source $i_{S}$. However, in most cases, circuits are driven by voltage sources. In this section, the circuit of Fig.7(c) driven by a voltage source, as it is shown in Fig.7(d), is proposed. The smooth cubic $i-v$ characteristic of the nonlinear resistor of the circuit of Fig.7(d) remains the same as in equation (31).

By applying the two Kirchhoff laws, the system of differential equations (36) is obtained:

$\left\{\begin{array}{l}\frac{\mathrm{d} x}{\mathrm{~d} \tau}=x(1-\varepsilon)-\frac{1}{3} x^{3}-y+z \\ \frac{\mathrm{d} y}{\mathrm{~d} \tau}=c(x+a-b y)\end{array}\right.$

where, $\quad \tau=\frac{t}{\rho C}, \quad x=\frac{v}{V_{0}}, \quad y=\frac{\rho i_{L}}{V_{0}}, \quad z=\frac{\rho v_{\mathrm{S}}}{R_{S} V_{0}}, \quad \alpha=\frac{E}{V_{0}}$, $b=\frac{R}{\rho}, c=\frac{\rho^{2} C}{L}$ and $\varepsilon=\frac{\rho}{R_{S}}$.

The driving voltage source has the following form:

$z=U_{0} \cos \left(2 \pi f_{N} \tau\right)$

where the normalized frequency is $f_{N}=\rho C f$. 
Ch. K. Volos, I. M. Kyprianidis, I. N. Stouboulos, and S. Vaidyanathan /

Journal of Engineering Science and Technology Review 8 (2) (2015) 157 - 173
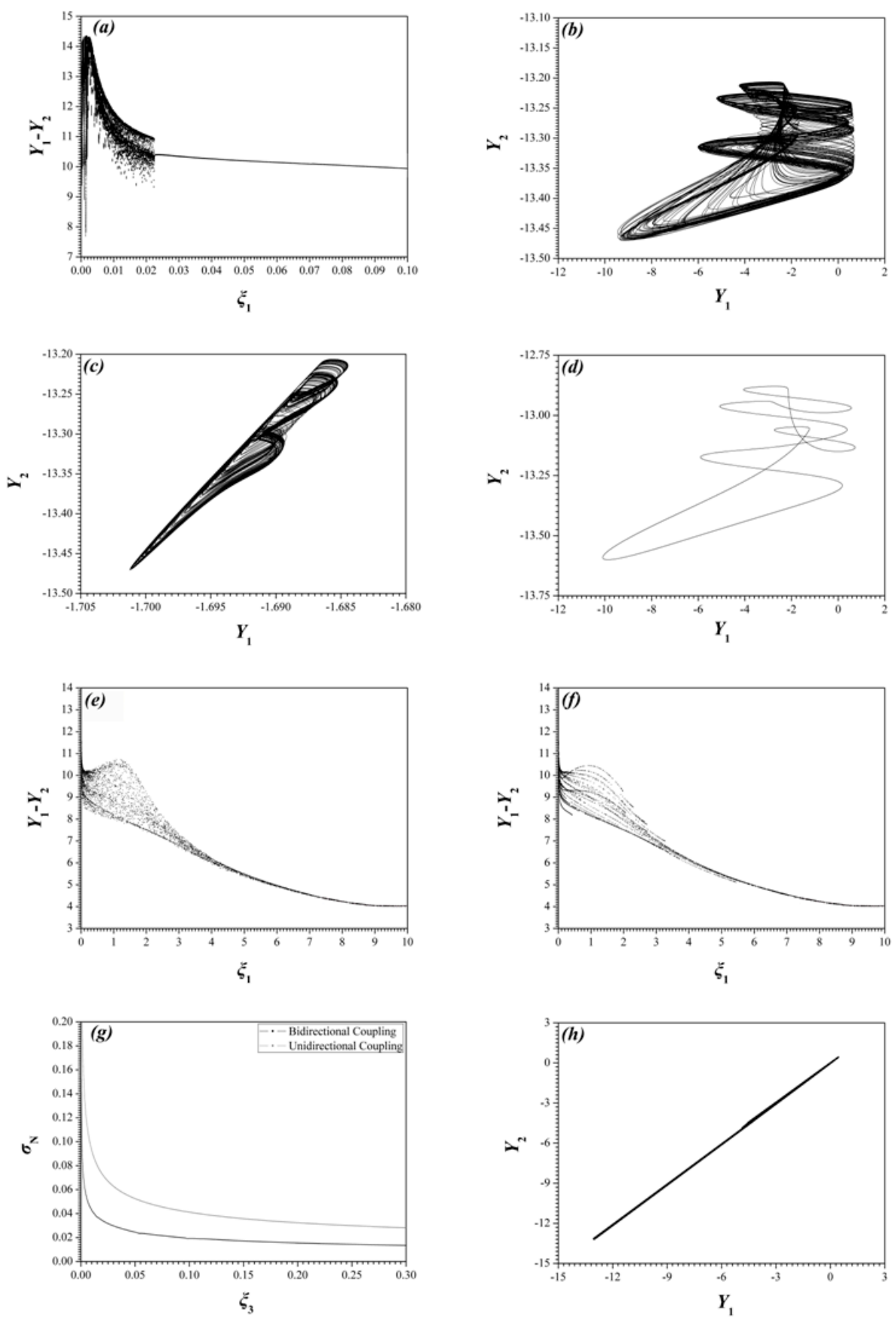

Fig. 6. (a) Bifurcation diagram of $\left(Y_{2}-Y_{1}\right)$ vs. $\xi_{1}$, in the case of mutually coupled neuron-cells, for $\xi_{3}=0$, (b) Phase portraits of $Y_{2}$ vs. $Y_{1}$, for $\xi_{3}=0$ and $\xi_{1}=0.02$, (c) Phase portraits of $Y_{2}$ vs. $X_{2}$, for $\xi_{3}=0$ and $\xi_{1}=0.02$ and (d) Phase portraits of $Y_{2}$ vs. $Y_{1}$, for $\xi_{3}=0$ and $\xi_{1}=0.05$; (e) \& (f) Bifurcation diagrams of $\left(Y_{2}-Y_{1}\right)$ vs. $\xi_{1}$, with the two different approaches, in the case of unidirectionally coupled neuron-cells, for $\xi_{3}=0$; (g) Diagram of the normalized standard deviation $\sigma_{N}$ vs. the parameter $\xi_{3}$, in the cases of bidirectionally (black line) and unidirectionally (gray line) coupling, for $\xi_{1}=0.02$; (h) Phase portrait of $Y_{2}$ vs. $Y_{1}$, for $\left(\xi_{1}, \xi_{3}\right)=(0.02,0.3)$. In all cases the following parameters have been used. $b=2.82, d=5.0$, $r=0.02, s=4.0, \chi=1.6$ and $f_{N}=0.16$, and initial conditions $\left(X_{1}, Y_{1}, Z_{1}, X_{2}, Y_{2}, Z_{2}, U\right)=(0.200,0.001,0.002,0.100,0.003,0.004,0.500)$.

For studying the way in which a stimulation signal affects the dynamic behavior of coupled, via a synapse, neuron's models of this type, a system of two unidirectionally coupled, via the aforementioned memristor, circuits of Figs.7(c) \& 7(d), which have identical circuit elements, $L, R, C, E$ and $N_{R}$, is proposed.

The first circuit models a neuron which is stimulated while the second is a neuron which receives, via the synapse, the transmitted signal. In this way of coupling only the second circuit is influenced by the first one. By applying the first Kirchhoff law to the second circuit, we obtain:

$$
i_{C 2}+i_{L 2}+i_{N 2}=i_{M}
$$

where,

$$
i_{M}=w(\varphi) v_{M}
$$


and $w(\varphi)$ the memductance provided by the equation (22). So, by using the previously introduced normalized variables and the normalized variable of the memristor, $u=\frac{\varphi}{V_{0} \rho C}$, the system of Fig.7(e) can be described by the following normalized system of differential equations (40).

$$
\left\{\begin{array}{l}
\frac{\mathrm{d} x_{1}}{\mathrm{~d} \tau}=x_{1}(1-\varepsilon)-\frac{1}{3} x_{1}^{3}-y_{1}+z \\
\frac{\mathrm{d} y_{1}}{\mathrm{~d} \tau}=c\left(x_{1}+a-b y_{1}\right) \\
\frac{\mathrm{d} x_{2}}{\mathrm{~d} \tau}=x_{2}-\frac{1}{3} x_{2}^{3}-y_{2}+\left(\xi_{1}+3 \xi_{3} u^{2}\right)\left(x_{1}-x_{2}\right) \\
\frac{\mathrm{d} y_{2}}{\mathrm{~d} \tau}=c\left(x_{2}+a-b y_{2}\right) \\
\frac{\mathrm{d} u}{\mathrm{~d} \tau}=x_{1}-x_{2}
\end{array}\right.
$$

where, $\xi_{1}=\rho k_{1}$ and $\xi_{3}=\rho^{3} V_{0}^{2} C^{2} k_{3}$.

Kyprianidis et al. [72, 73] showed that the first subcircuit of the proposed coupled system (Fig.7(e)) can operate in a chaotic mode for specific values of system's parameters and initial conditions. Figs.8(a) \& 8(b) display the phase portrait of $y_{1}$ versus $x_{1}$ and the time-series of signal $x_{1}$, which confirm the expected chaotic behavior of the first uncoupled cell, for $a=0.70, b=0.80, c=0.10, f_{N}=0.16, \varepsilon=0.16$ and $U_{0}=0.9$.

At the beginning, for studying the effect, of the coupling on system's behavior, the parameter $\xi_{3}$ remains equal to zero, while $\xi_{1}$ takes various values, in the interval $[0,10]$. In this case, the two cells are linearly coupled, so the memristor simulates a gap junction between the two neuron-cells. The bifurcation diagram of the signal difference $\left(y_{1}-y_{2}\right)$, with respect to the bifurcation parameter $\xi_{1}$ (Figure 8(c)) is used, for studying analytically the dynamic behavior of the system. As it is shown, for low values of $\xi_{1}$ the system is in a chaotic state (Fig.8(d)), the range of which is gradually narrowed and ultimately results in a complete chaotic synchronization mode (Fig.8(e)).

So, the gap junction controls the flow of energy between the two neuron-cells and as the coupling coefficient $\xi_{1}$ increases, it suppresses the chaotic state of the system.

Next, in order to study the effect of the second term of the memductance and consequently of the specific type of memristor to system's behavior, the parameter $\xi_{3}$ is changed. From the bifurcation diagram of $\left(y_{2}-y_{1}\right)$ versus the memristor's parameter $\xi_{3}$ (Fig.8(f)), one could see that the system is driven from the desynchronization to complete synchronization almost immediately for extremely low values of $\xi_{3}$. In more detail, as it is displayed in Figs.8(g) \& 8(h) the two coupled neuronal cells remain in a desynchronization state for low values of $\xi_{3}\left(\xi_{3}=0.0001\right)$, while for greater values of $\xi_{3}\left(\xi_{3}=0.1\right)$ the coupled neuronal cells are synchronized and remain in this steady state as the $\xi_{3}$ is further increased. So, the proposed memristor contributes to the chaotic synchronization of the coupled system of neuron-cells. Therefore, in this case the memristor controls again the flow of energy between the two neuroncells and provides greater suppress to the chaotic state of the system, leading it to the complete synchronization state.
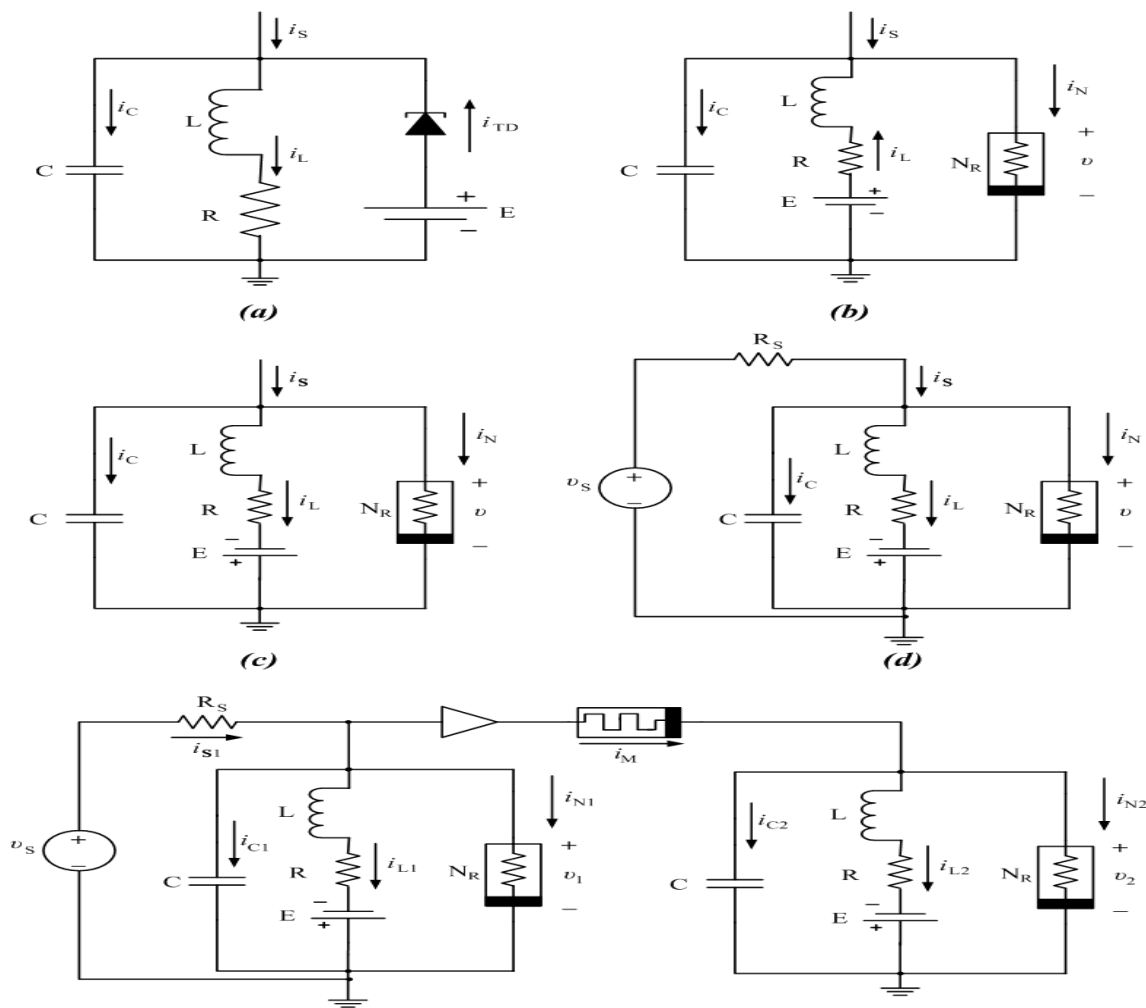

(e)

Fig. 7. (a) The electronic simulator of the BvP model proposed by Nagumo et al.; (b) The electronic simulator of the BvP model of FitzHugh model, proposed in the present work; (c) The nonlinear electric circuit simulating system (33); (d) The equivalent circuit of BvP oscillator's state equations by Rajasekar and Lakshmanan driven by a voltage source; (e) The coupled system via the proposed memristor. 

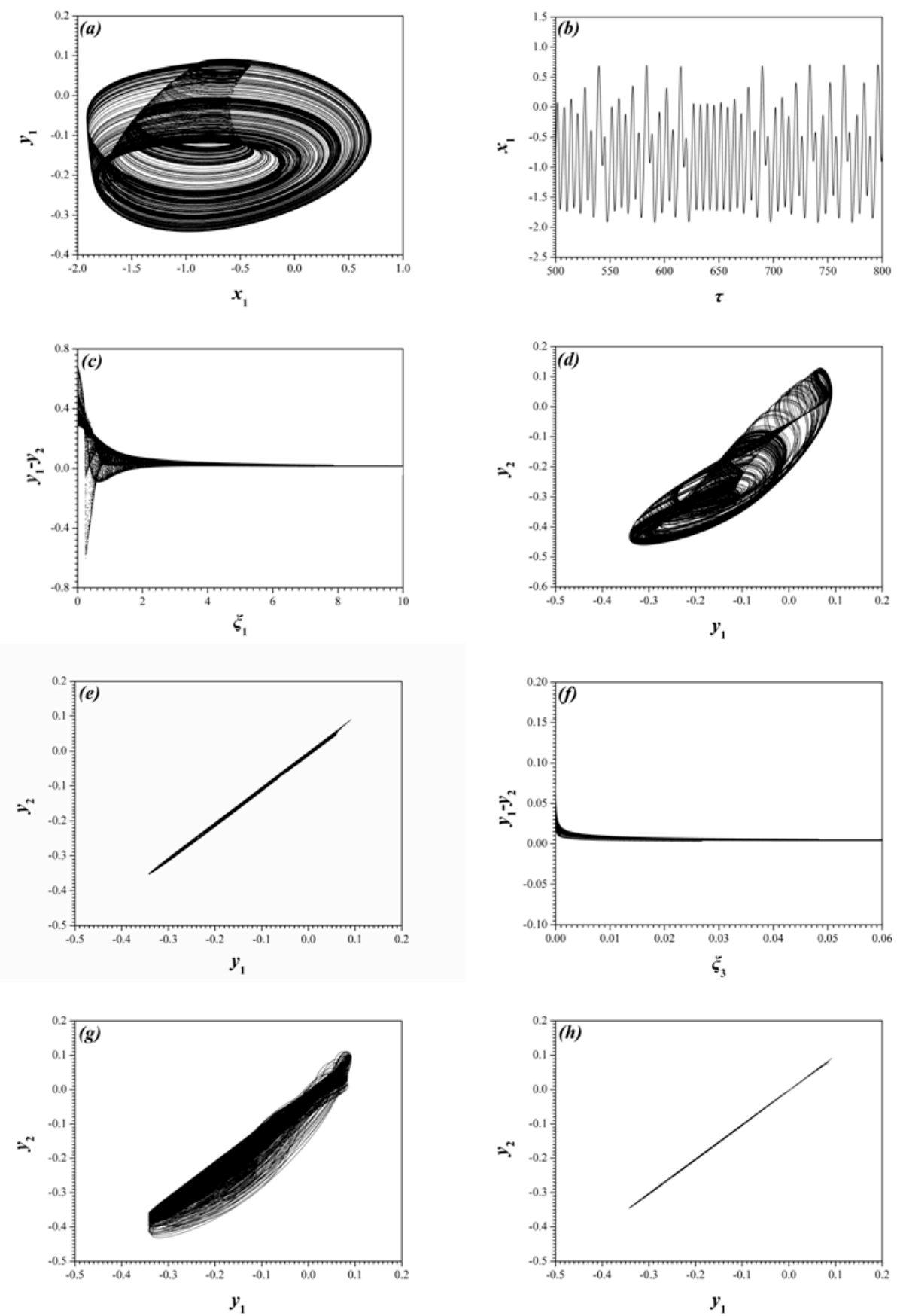

Fig. 8. (a) \& (b) Phase portrait of $y_{1}$ vs. $x_{1}$ and the time-series of signal $x_{1}$, which confirm the expected chaotic behavior of the first neuron-cell; (c) Bifurcation diagram of $\left(y_{1}-y_{2}\right)$ vs. $\xi_{1}$, in the case of coupled neuron-cells, for $\xi_{3}=0$; (d) \& (e) Phase portraits of $y_{2}$ vs. $y_{1}$, for $\xi_{3}=0$ and $\xi_{1}=1$, $\xi_{1}=10$; (f) Bifurcation diagram of $\left(y_{1}-y_{2}\right)$ vs. $\xi_{3}$, in the case of coupled neuron-cells, for $\xi_{1}=1$; (g) \& (h) Phase portraits of $y_{2}$ vs. $y_{1}$, for $\left(\xi_{1}, \xi_{3}\right)=(1,0.001)$ and $\left(\xi_{1}, \xi_{3}\right)=(1,0.1)$. In all cases the following parameters and initial conditions have been used. $a=0.70, b=0.80, c=0.10$, $f_{N}=0.16, \varepsilon=0.16, U_{0}=0.9,\left(x_{1}, y_{1}, x_{2}, y_{2}, u\right)=(0.5,0.1,0.8,0.4,0.5)$.

\section{Conclusion}

This paper was based on the latest developments in the very interesting field of memristors and their potential applications. In this sense, a series of promising applications in analog and digital circuits design or in other scientific fields was presented. However, probably the most interesting application of memristor, due to its nature, is in neuromorphic circuits.

As it was referred within the paper, the last five years, many research teams have presented various types of memristor and techniques which were used for studying the case-study of using this element as an artificial synapse. Among the recorded in the literature different approaches on this subject, a flux-controlled memristor of cubic $\varphi-q$ function, for emulating the excitatory and inhibitory synaptic connection as well as the ohmic connections was adopted.

Therefore, for the purposes of this work, two of the most well-known neuron models, the Hindmarsh- Rose and FitzHugh-Nagumo, which were coupled via the proposed memristor, were chosen. In this direction, electronic circuits which simulate the dynamic behavior of the aforementioned neuron models were designed.

The intensively study of the coupling schemes 
(bidirectional and unidirectional), by using tools of nonlinear dynamics, such as the bifurcation diagram and the phase portrait, revealed the rich dynamic behavior of the coupled systems. Interesting phenomena, depending on the memristor's parameters values, were revealed. Chaotic behavior for extremely small values of the parameters, periodic states and synchronization mode of the coupled neuron-cells, were studied. Nevertheless, synchronization was the most interesting phenomenon because it was shown that the proposed memristor could be used as an artificial synapse for transmitting information between interconnected neuron-cells.

\section{References}

1. L.O. Chua, Memristor - the missing circuit element, IEEE Transactions on Circuit Theory, vol. 18(5), pp. 507-519 (1971).

2. L.O. Chua and S.M. Kang, Memristive devices and systems, In Proceedings of the IEEE, vol 64, pp. 209-223 (1976).

3. C. Sanchez-Lopez, J. Mendoza-Lopez, M.A. Carrasco-Aguilar, and C. Muniz-Montero, A floating analog memristor emulator cCircuit, IEEE Trans on Circuits and Systems II-Express, vol. 61(5), pp. 309-313 (2014).

4. D. Biolek, M. Di Ventra, and Y.V. Pershin, Reliable SPICE simulations of memristors, memcapacitors and meminductors, Radioengineering, vol. 22(4), pp. 945-968 (2013).

5. Z. Kolka, D. Biolek, and V. Biolkova, Frequency-domain steady-state analysis of circuits with mem-elements, Analog Integrated Circuits and Signal Processing, vol. 74(1), pp. 79-89 (2013).

6. D. Strukov, G. Snider, G. Stewart, and R. Williams, The missing memristor found, Nature, vol. 453, pp. 80-83 (2008).

7. M.D. Pickett, R.S. Williams, Sub-100 fJ and sub-nanosecond thermally driven threshold switching in niobium oxide crosspoint nanodevices, Nanotechnology, vol. 23, p. 215202 (2012).

8. M. Sapoff and R.M. Oppenheim, Theory and application of selfheated thermistors, In Proceedings of the IEEE, vol 51, pp. 1292-1305 (1963)

9. Y.V. Pershin and M. Di Ventra, Spin memristive systems: Spin memory effects in semiconductor spintronics, Physics Review B, vol. 78, pp. 113309/1-4 (2008).

10. Y. Chen, G.Y. Jung, D.A. A. Ohlberg, X.M. Li, D.R. Stewart, J.O. Jeppesen, K.A. Nielsen, J.F. Stoddart, and R.S. Williams, Nanoscale molecular-switch crossbar circuits, Nanotechology, vol. 14, pp. 462-468 (2003).

11. Y.V. Pershin and M. Di Ventra, Experimental demonstration of associative memory with memristive neural networks, Neural Networks, vol. 23, p. 881 (2010).

12. Y.V. Pershin, S. La Fontaine, and M. Di Ventra, Memristive model of amoeba learning, Physics Review E, vol. 80, pp. 021926/1-6 (2009).

13. L.O. Chua, Memristor, Hodgkin-Huxley, and Edge of Chaos, Nanotechnology, vol. 24, pp. 383001/1-14 (2013).

14. S.H. Jo, T. Chang, I. Ebong, B.B. Bhadviya, P. Mazumder, and W. Lu, Nanoscale memristor device as synapse in neuromorphic systems, Nano Letters, vol. 10, pp. 1297-1301 (2010).

15. M. Laiho and E. Lehtonen, Cellular nanoscale network cell with memristors for local implication logic and synapses, In Proceedings IEEE of International Symposium on Circuits and Systems (ISCAS 2010), pp. 2051-2054 (2010).

16. B. Linares-Barranco and T. Serrano-Gotarredona, Memristance can explain spike-time-dependent-plasticity in neural synapses, In Proceedings of Nature, pp. 1-4 (2009).

17. M. Itoh and L.O. Chua, Memristor oscillators, International Journal of Bifurcation and Chaos, vol. 18, pp. 3183-3206 (2008).

18. B. Muthuswamy and P.P. Kokate, Memristor based chaotic circuits, IETE Technical Review, vol. 26, pp. 415-426 (2009).

19. B. Muthuswamy, Implementing memristor based chaotic circuits, International Journal of Bifurcation and Chaos, vol. 20, pp. 1335-1350 (2010).

20. D.B. Strukov and R.S. Williams, Four-dimensional address topology for circuits with stacked multilayer crossbar arrays, In Proceedings of the National Academy of Science USA, vol. 106, pp. 20155-20158 (2009).

21. P. Vontobel, W. Robinett, J. Straznicky, P.J. Kuekes, and R.S. Williams, Writing to and reading from a nano-scale crossbar memory based on memristors, Nanotechnology, vol. 20, pp. 425204/1-21 (2009).
22. F. Zhuge, W. Dai, C.L. He., A.Y. Wang, Y.W. Liu, M. Li, Y.H. $\mathrm{Wu}, \mathrm{P}$. Cui, and R.W. Li, Nonvolatile resistive switching memory based on amorphous carbon, Applied Physics Letters, vol. 96, pp. 163505-163507 (2010).

23. Y.V. Pershin and M. Di Ventra, Practical approach to programmable analog circuits with memristors, IEEE Transactions on Circuits and Systems I, vol. 57, pp. 1857-1864 (2010).

24. T. Driscoll, J. Quinn, S. Klein, H.T. Kim, B.J. Kim, Y.V. Pershin, M. Di Ventra, and D.N. Basov, Memristive adaptive filters, Applied Physics Letters, vol. 97, pp. 093502/1-3 (2010).

25. J. Clarke and F.K. Wilhelm, Superconducting quantum bits, Nature, vol. 453, pp. 1031-1042 (2008).

26. A. Zagoskin and A. Blais, Superconducting qubits, Physics in Canada, vol. 63, p. 215 (2007).

27. Y.V. Pershin and M. Di Ventra, Neuromorphic, digital and quantum computation with memory circuit elements, arXive: 1009.6025 (2010).

28. Q. Xia, W. Robinett, M.W. Cumbie, N. Banerjee, T. J. Cardinali, J.J. Yang, W. Wu, X. Li, W.M. Tong, D.B. Strukov, G.S. Snider, G. Medeiros-Ribeiro, and R.S. Williams, Memristor-CMOS hybrid integrated circuits for reconfigurable logic, Nano Letters, vol. 9, pp. 3640-3645 (2009).

29. J. Borghetti, Z. Li, J. Straznicky, X. Li, D. A. A. Ohlber, W. Wu, D.R. Stewart, and R.S. Williams, A hybrid nanomemristor/transistor logic circuit capable of selfprogramming, In Proceedings of the National Academy of Science USA, vol. 106, pp. 1699-1703 (2009).

30. E. Lehtonen, J.H. Poikonen, M. Laiho, Two memristors suffice to compute all Boolean functions, Electronics Letters, vol. 46, pp. 239-240 (2010).

31. T. Driscoll, H.-T. Kim, B.-G. Chae, B.-J. Kim, Y.-W. Lee, N.M. Jokerst, S. Palit, D.R. Smith, M. Di Ventra, and D.N. Basov, Memory metamaterials, Science, vol. 325, pp. 1518-1521 (2009).

32. S. Shin, K. Kim, and S.-M.S. Kang, Memristor applications for programmable analog ICs, IEEE Transactions on Nanotechnology, vol. 10, pp. 266-274 (2010).

33. T. Prodromakis and C. Toumazou, A review on memristive devices and applications, In Proceedings of IEEE International Conference on Electronics, Circuits, and Systems, pp. 934-937 (2010).

34. M.P. Kennedy, Three steps to chaos II. A Chua's circuit primer, IEEE Transactions on Circuits \& Systems - Part 1, vol. 40, pp. 657-674 (1993).

35. M. Itoh and L.O. Chua, Duality of memristor circuits, International Journal of Bifurcation and Chaos, vol. 23, pp. 133000/1-50 (2013).

36. B. Muthuswamy and L.O. Chua, Simplest chaotic circuit, International Journal of Bifurcation and Chaos, vol. 20, pp. 1567-1580 (2010).

37. B. Bo-Cheng, L. Zhong and X. Jian-Ping, Transient chaos in smooth memristor oscillator, Chinese Physics B, vol. 19, pp. 030510/1-6 (2010).

38. L.O. Chua, Resistance switching memories are memristors, Applied Physics A, vol. 102, pp. 765-783 (2011).

39. I.M. Kyprianidis, Ch.K. Volos, and I.N. Stouboulos, Chaotic dynamics from a nonlinear circuit based on memristor with cubic nonlinearity, In Proceedings of 7 th International Conference of the Balkan Physical Union, AIP Conference Proceedings, vol. 1203, pp. 626-631 (2010).

40. Ch.K. Volos, I.M. Kyprianidis, S.G. Stavrinides, I.N. Stouboulos, and A.N. Anagnostopoulos, Memristors: A new approach in nonlinear circuits design, In Proceedings of 14th 
WSEAS International Conference on Communications, pp. 2530 (2010).

41. A. Mittal and S. Swaminathan, Image stabilization using memristors, In Proceedings IEEE of 2nd International Conference on Mechanical and Electrical Technology (ICMET) (2010).

42. Z. Lin and H. Wang, Efficient image encryption using a chaosbased pwl memristor, IETE Technical Review, vol. 27, pp. 318325 (2010).

43. E Schulte, U. Schumacher, and J. Rude, Thieme atlas of anatomy: Head and neuroanatomy, Thieme, New York, USA (2007).

44. E.M. Izhikevich, Neural Excitability, spiking and bursting, International Journal of Bifurcation and Chaos, vol. 10, pp. 1171-1266 (2000).

45. L. Sherwood, Human Physiology: From Cells to Systems, Brooks/Cole, Cengage Learning, Belmont, USA (2013)

46. A.L. Hodgkin and A.F. Huxley, A quantitative description of membrane current and its application to conduction and excitation in nerve, Journal of Physiology-London, vol. 117, pp. 500-544 (1952).

47. R. FitzHugh, Impulses and physiological states in theoretical models of nerve membrane, Biophysical Journal, vol. 1, pp. 445466 (1961).

48. J.L. Hindmarsh and R.M. Rose, A model of neuronal bursting using three coupled first order differential equations, In Proceedings of Royal Society London, Series B, vol. 221, pp. 87-102 (1984).

49. A.V.M. Herz, T. Gollisch, C.K. Machens, and D. Jaeger, Modeling single-neuron dynamics and computations: A balance of detail and abstraction, Science, vol. 314, pp. 80-85 (2006).

50. G.M. Shepherd, Neurobiology, Oxford University Press, Oxford, New York (1994).

51. A. Afifi, A. Ayatollahi, and F. Raissi, Implementation of biologically plausible spiking neural network models on the memristor crossbar-based CMOS/nano circuits, In Proceedings IEEE of the European Conference on Circuit Theory and Design, (ECCTD 2009), pp. 563-566 (2009).

52. M.J. Sharifi and Y.M. Banadaki, General spice models for memristors and application to circuit simulation of memristorbased synapses and memory cells, Journal of Circuits, Systems and Computers, vol. 19, pp. 407-424 (2010)

53. H. Kim, M.P. Sah, C. Yang, T. Roska, and L.O. Chua, Neural synaptic weighting with a pulse-based memristor circuit, IEEE Transactions Circuits and Systems I: Regular Papers, vol. 59, pp. 148-158 (2012).

54. M.P. Sah, C. Yang, H. Kim, and L.O. Chua, A voltage mode memristor bridge synaptic circuit with memristor emulators, Sensors, vol. 12, pp. 3587-3604 (2012).

55. B. Bo-Cheng, X. Jian-Ping, and L. Zhong, Initial state dependent dynamical behaviors in a memristor based chaotic circuit, Chinese Physics Letters, vol. 27, pp. 070504/1-3 (2010).

56. Ch.K. Volos, I.M. Kyprianidis, and I.N. Stouboulos, The memristor as an electric synapse - synchronization phenomena, In Proceedings IEEE of Digital Signal Processing (DSP11), pp. 1-4 (2011).
57. G.S. Snider, Spike-timing-dependent learning in memristive nanodevices, In Proceedings of IEEE International Symposium on Nanoscale Architectures, (NANOARCH 2008), pp. 85-92 (2008).

58. D. Purves, G.J. Augustine, D. Fitzpatrick, L.C. Katz, A.S. LaMantia, J.O. McNamara, and S.M. Williams, Neuroscience, Sinauer Associates, Sunderland, UK (2001).

59. E. Mosekilde, Y. Maistrenko, and D. Postnov, Chaotic synchronization: Applications to living systems, World Scientific Publishing, Singapore (2002).

60. A.S. Pikovsky, M. Rosenblum, and J. Kurths, Synchronization: A universal concept in nonlinear sciences, Cambridge University Press, Cambridge, UK (2003).

61. H.D.I. Abarbanel, R. Huerta, M.I. Rabinovich, P.F. Rowat, N.F. Rulkov, and A.I. Selverston, Synchronized Action of synaptically coupled chaotic model neurons, Neural Computation, vol. 8, pp. 1567-1602 (1996).

62. L. Glass and M.C. Mackey, From clocks to chaos: The rhythms of life, Princeton University Press, Princeton, USA (1988).

63. M.I. Rabinovich and H.D.I. Abarbanel, The role of chaos in neural systems, Neuroscience, vol. 87, pp. 5-14 (1998).

64. H. Fujisaka and T. Yamada, Stability theory of synchronized motion in coupled oscillator systems, Progress of Theoretical Physics, vol. 69, pp. 32-47 (1983).

65. A.S. Pikovsky, On the interaction of strange attractors, Zeitschrift für Physik B - Condensed Matter, vol. 55, pp. 149154 (1984).

66. Ch.K. Volos, I.M. Kyprianidis, and I.N. Stouboulos, Experimental synchronization of two resistively coupled Duffing - type Circuits, Nonlinear Phenomena in Complex Systems, vol. 11, pp. 187-192 (2008).

67. Ch.K. Volos, I.M. Kyprianidis, and I.N. Stouboulos, A gallery of synchronization phenomena in resistively coupled nonautonomous chaotic circuits, Journal of Engineering Science and Technology Review, vol. 6(4), pp. 15-23 (2013).

68. C. Grebogi, S.W. McDonald, E Ott,.and J.A. Yorke, Final state sensitivity: An observation to predictability, Physics Letters A, vol. 99, pp. 415-418 (1983).

69. J. Nagumo, S. Arimoto, and S. Yoshizawa, An active pulse transmission line simulating Nerve axon, In Proceedings of IRE, vol. 50, pp. 2061-2070 (1962).

70. S. Rajasekar and M. Lakshmanan, Period-doubling bifurcations, chaos, phase-locking and devil's staircase in a Bonhoeffer - van der Pol oscillator, Physica D, vol. 32, pp. 146-152 (1988).

71. S. Rajasekar and M. Lakshmanan, Algorithms for controlling chaotic motion: Application for the BVP oscillator, Physica D, vol. 67, pp. 282-300 (1993).

72. I.M. Kyprianidis, V. Papachristou, I.N. Stouboulos, and Ch.K. Volos, Dynamics of coupled chaotic Bonhoeffer-van der Pol oscillators, WSEAS Transactions on Systems, vol. 11, pp. 516526 (2012).

73. I.M. Kyprianidis and A. T. Makri, Complex dynamics of FitzHugh-Nagumo type neurons coupled with gap junction under external voltage stimulation, Journal of Engineering Science and Technology Review, vol. 6(4), pp. 104-114 (2013). 